\title{
A Case of Linear Alopecia of the Scalp
}

\author{
Cecília Anatriello dos Santos ${ }^{\mathrm{a}}$ Andrezza Telles Westin ${ }^{\mathrm{a}}$ \\ Fernanda André Martins Cruz Perecin ${ }^{a}$ João Carlos Lopes Simão ${ }^{a}$ \\ Francesca Maia Faria ${ }^{b}$ Marco Andrey Cipriani Frade ${ }^{a}$
}

aDivision of Dermatology, Department of Internal Medicine, Hospital of the Clinics of Ribeirão Preto, University of São Paulo, Ribeirão Preto, São Paulo, Brazil; ' ${ }^{2}$ Department of Pathology, Hospital of the Clinics of Ribeirão Preto, University of São Paulo, Ribeirão Preto, São Paulo, Brazil

\section{Question}

A 14-year-old Caucasian male patient came to the health service, with a history of 1-year linear pattern hair loss (Fig. 1). Upon physical examination, we observed erythematous-violaceous plaque infiltrated in the buccinator region and a linear band of balding of approximately 20 $\mathrm{mm}$ width, distributed in the temporoparietal region. The dermoscopy scan evidenced the presence of follicular units with only 1 hair and mild scalp desquamation. There were no black or white dots as well as any exclamation mark hairs or broken hair. We found multiple yellow dots of

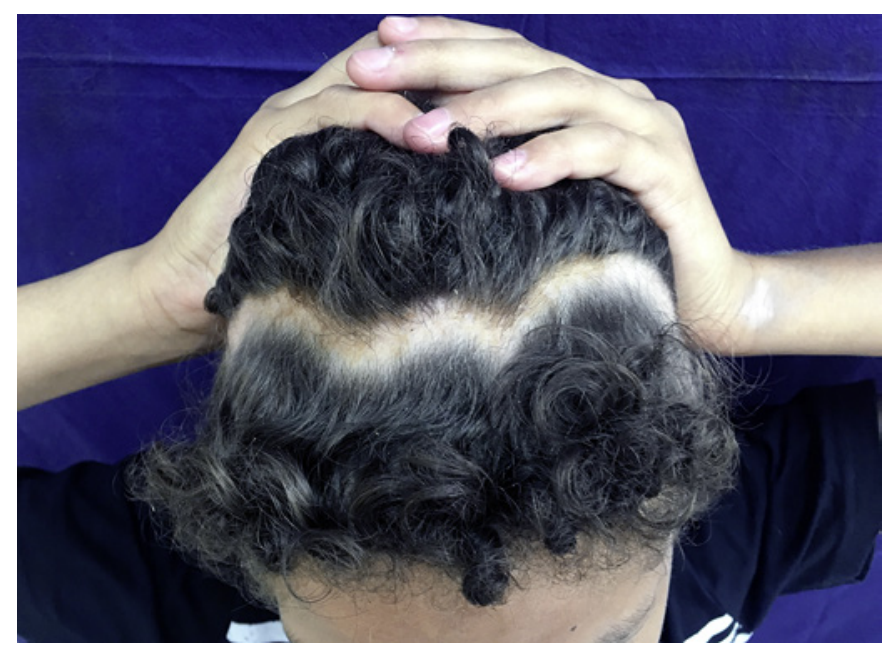

Fig. 1. Linear alopecia of wave and curved pattern. varying size (Fig. 2), few angulated hair and circle hair, several short regrowing vellus hairs, and rare dystrophic hairs. Prominent polymorphous blood vessels were the most frequent finding (Fig. 3). The rest of the physical examination was unremarkable. The patient did not present other pathologies or symptoms and denied use of hair bands or any other accessory that could compress the region.

Histopathological examination of a biopsy from the scalp revealed epidermis with no changes, dermis with perivascular and periadnexal lymphocyte inflammatory infiltration. The most important findings were at the subcutaneous fat tissue, in which we observed lobular panniculitis with predominantly lymphohistiocytic inflammatory infiltrate (Fig. 4), subcutaneous mucin deposition. It also demonstrated an increased percentage of the catagen/telogen to anagen ratio. The immunofluorescence was negative. Otherwise, facial lesion analysis revealed epidermal atrophy, hydropic degeneration of the basal layer, perivascular, and periadnexal lymphocyte inflammatory infiltration. Serological tests (ANA, anti-Ro, anti-La, anti-SM/RNP, native anti-DNA, complement dosage, and blood count) showed no changes. Systemic involvement was not observed, either.

After clinical and histopathological correlation, the diagnosis was confirmed. Specific therapy initiated and the patient evolved with total hair regrowth (Fig. 5). He has been under dermatological follow-up for 2 years, with no recurrences.

What is your diagnosis?

$\begin{aligned} & \text { karger@karger.com } \\ & \text { www.karger.com/sad }\end{aligned}$
Karger ${ }^{\prime /}$

Cecília Anatriello dos Santos

Division of Dermatology, Department of Internal Medicine Hospital of Clinics of Ribeirão Preto, University of São Paulo Avenida Bandeirantes, 3900, Ribeirão Preto, SP 14015-010 (Brazil) ceciliaasantos@yahoo.com.br 
Fig. 2. Trichoscopy $(\times 20)$ showing short regrowing vellus hairs (white arrow), multiple yellow dots of varying size (black arrow), and angulated hair (red arrow).

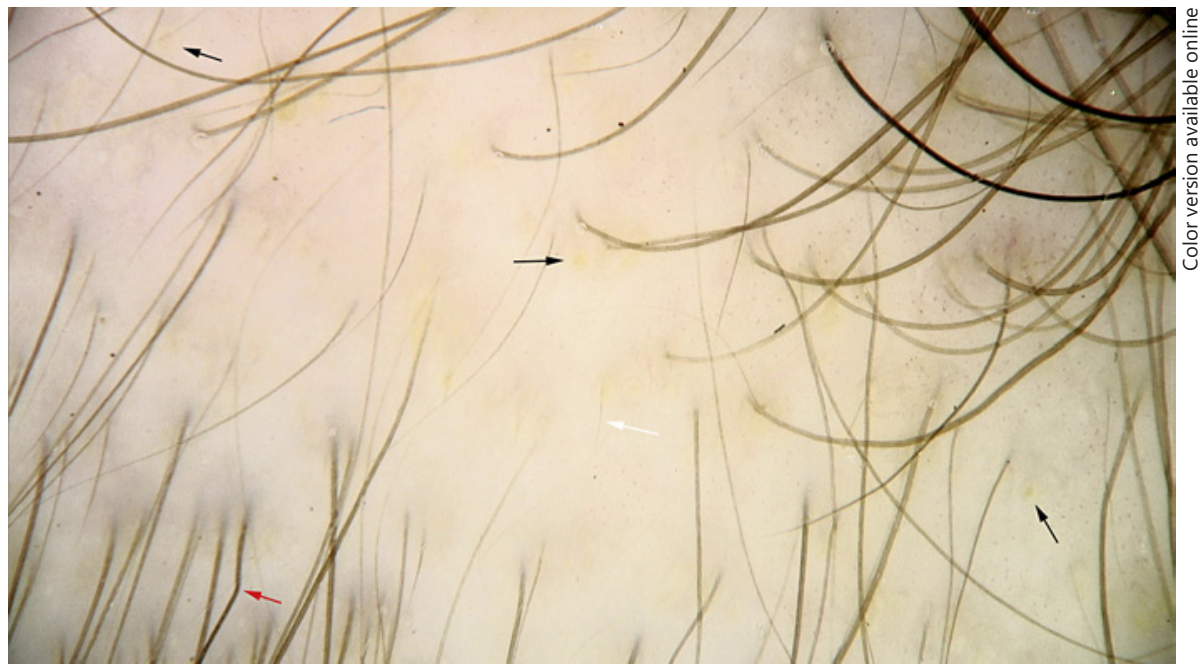

Fig. 3. Trichoscopy $(\times 20)$ showing dystrophic hairs (black arrow) and prominent polymorphous blood vessels (red arrow).

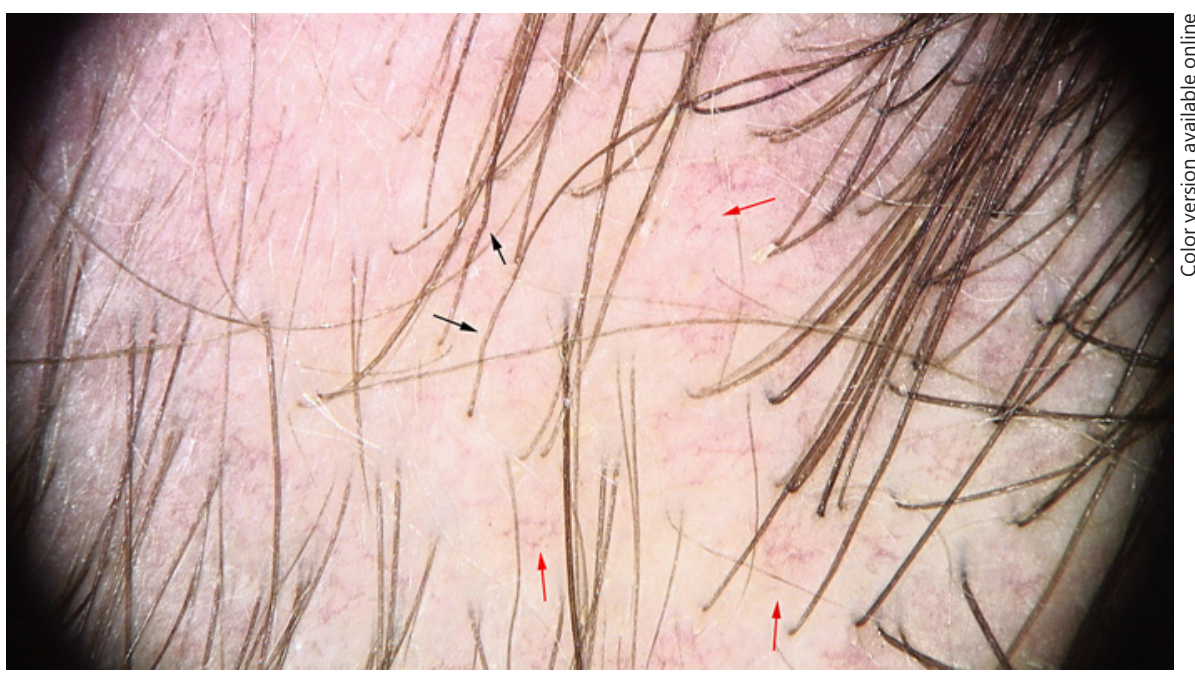

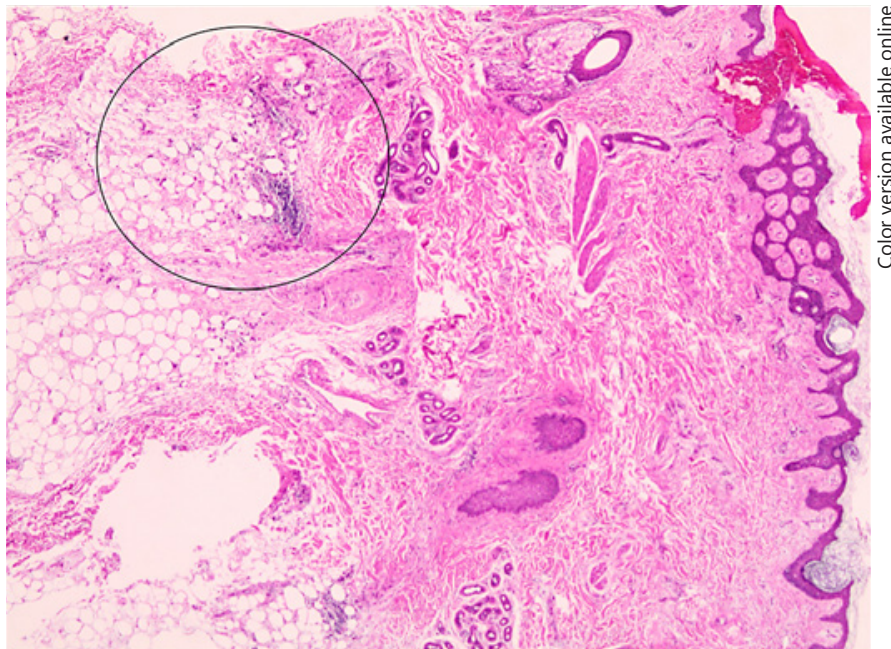

Fig. 4. Lobular panniculitis (black circle) with predominantly lymphohistiocytic inflammatory infiltrate (histopathology findings of scalp biopsy, $\times 40)$. H\&E.

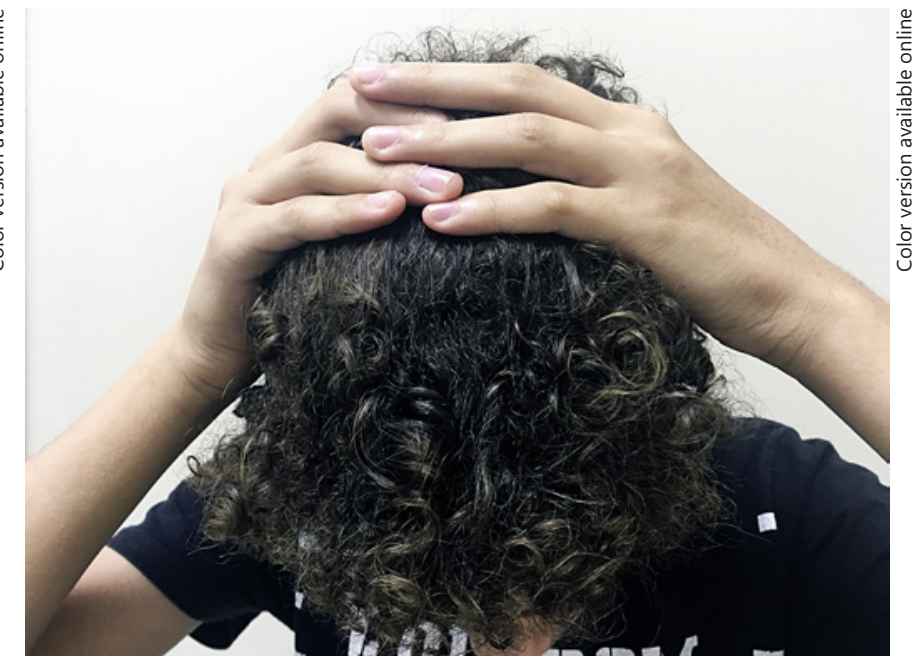

Fig. 5. Complete hair regrowth of the linear alopecia. 


\section{Answer}

The patient's diagnosis, according to the clinical case and histopathological findings, was lupus panniculitis of the scalp (LPS)-associated chronic cutaneous lupus erythematosus of the face. The patient was treated with methotrexate (15 mg per week) and prednisolone (40 mg per day), showing complete hair regrowth and total disappearance of the facial lesion within 4 months of treatment. The patient self-suspended the treatment after 10 months, remaining without relapses for 2 years.

LPS has peculiarities that differentiate it from classic lupus panniculitis. LPS affects younger patients, is less associated with systemic involvement, and has a reversible clinical course [1]. The feature that most differentiates LPS from classic lupus panniculitis is its non-scarring nature [2]. Moreover, there is lower frequency of anti-nucleus factor positivity [3]. Also, the lesions frequently occur in linear, arciform, or annular arrangement along the Blaschko's lines $[3,4]$. A recent review by Lueangarun et al. [3] described that the most commonly affected area of scalp is the parietal site (70\%); however, the alopecia may involve all areas of the scalp.

The recent report of Udompanich et al. [2] described insight into the trichoscopic features of LPS, including broken hair, exclamation mark hair, angulated hair, and short regrowing hair, suggesting an inflammatory insult to the hair bulb leading to increased hair shaft fragility. In addition to variable sizes, yellow dots are sparsely arranged [2]. The differential diagnoses with clinical and dermoscopic examination of the linear non-scarring alopecia, including alopecia areata, trichotillomania, and traction alopecia $[3,5]$.

Histopathological findings of inflammatory changes predominantly in the subcutaneous adipose tissue are suggested as probably responsible for the deregulation of the follicular growth cycle since the capillary bulbs of the anagen follicles are found in this area [6]. In addition, mucin deposition rich in glycosaminoglycans may lead to inhibitor effects in hair growth. Pro-inflammatory cytokines (interferon gamma) and lymphocytic infiltration would act as inducers for the catagen phase explained by the hypothesis of a synchronized and intense attack with predilection to the anagen follicles, as that occurs in other autoimmune alopecia, as alopecia areata [7-10].

The pathogenesis related to the Blaschko's line distribution is not clear. Mutations are believed to occur during embryogenesis leading to the formation of cellular mosaicism, with abnormal cell clones. These clones would not produce initial clinical signs but predispose to local inflammatory activation later, by exposing new antigens in susceptible patients [4].

In addition, LPS shows rapid response to treatment (about 8 weeks) [3] without the occurrence of scarring alopecia. Nevertheless, recurrences are frequent. In conclusion, we report a case of linear non-scarring alopecia along Blaschko's line with trichoscopic findings as described in the literature and biopsy results consistent with lupus panniculitis which had rapid response to established therapy resulting in complete hair regrowth.

\section{Acknowledgement}

We thank the members of the division of Dermatology and Department of Pathology of Hospital of Clinics of Ribeirao Preto.

\section{Statement of Ethics}

The study was approved by the Ethics Committee of our Hospital. The patient's mother gave written informed consent to publish the photos and the details of the case.

\section{Conflict of Interest Statement}

The authors have no conflicts of interest to declare.

\section{Funding Sources}

No funding sources to this report.

\section{Author Contributions}

Cecília Anatriello dos Santos: patient care, article making, and bibliographic review. Andrezza Telles Westin: patient care, bibliographic review, and article review. Fernanda André Martins Cruz Perecin: patient care, bibliographic review, and article review. João Carlos Lopes Simão: patient care and article review. Francesca Maia Faria: anatomopathological analysis. Marco Andrey Cipriani Frade: article review.

\section{Keywords}

Lupus panniculitis - Lupus panniculitis of the scalp . Alopecia $\cdot$ Scalp disease 


\section{References}

1 Chen YA, Hsu CK, Lee JYY, Yang CC. Linear lupus panniculitis of the scalp presenting as alopecia along Blaschko's lines: a distinct variant of lupus panniculitis in East Asians? J Dermatol. 2012;39:385-8.

2 Udompanich S, Chanprapaph K, Suchonwanit $P$. Linear and annular lupus panniculitis of the scalp: case report with emphasis on trichoscopic findings and review of the literature. Case Rep Dermatol. 2019 Jun 6;11(2): 157-65.

3 Lueangarun S, Subpayasarn U, Tempark T. Distinctive lupus panniculitis of scalp with linear alopecia along Blaschko's lines: a review of the literature. Int J Dermatol. 2019 Feb;58(2):144-50.
4 Lueangarun S, Subpayasarn U, Chakavittumrong $\mathrm{P}$, Tempark T, Suthiwartnarueput $\mathrm{W}$. Lupus panniculitis of the scalp presenting with linear alopecia along the lines of Blaschko. Clin Exp Dermatol. 2017 Aug;42(6):7057.

5 Park SK, Yoo HH, Yun SK, Kim HU, Park J. Geometric alopecia associated with lupus erythematosus panniculitis of the scalp: a case series of nine Korean patients. Eur J Dermatol. 2018;28(3):399.

6 Madaan A, Verma R, Singh AT, Jaggi M. Review of hair follicle dermal papilla cells as in vitro screening model for hair growth. Int J Cosmet Sci. 2018;40(5):429-50.
7 Coulson-Thomas VJ, Gesteira TF, Esko J, Kao W. Heparan sulfate regulates hair follicle and sebaceous gland morphogenesis and homeostasis. J Biol Chem. 2014;289(36):25211.

8 Paus R. Hair growth inhibition by heparin in mice: a model system for studying the modulation of epithelial cell growth by glycosaminoglycans? Br J Dermatol. 1991;124(5):415.

9 Harries MJ, Meyer KC, Paus R. Hair loss as a result of cutaneous autoimmunity: frontiers in the immunopathogenesis of primary cicatricial alopecia. Autoimmun Rev. 2009;8(6): 478 .

10 Udompanich S, Chanprapaph K, Suchonwanit P. Hair and scalp changes in cutaneous and systemic lupus erythematosus. Am J Clin Dermatol. 2018;19(5):679. 